\title{
La métrologie de la dose au CEA : le Laboratoire National Henri Becquerel
}

\author{
J. BARTHE ${ }^{1}$, B. CHAUVENET ${ }^{1}$, J.-M. BORDY ${ }^{1}$
}

(Manuscrit reçu le 6 novembre 2006, accepté le 28 février 2007)

RÉSUMÉ On présente un bref exposé des activités de métrologie de la dose du Laboratoire National Henri Becquerel (LNE-LNHB), laboratoire national de métrologie pour les rayonnements ionisants. Les résultats des principaux travaux effectués ces dernières années dans ce domaine sont exposés, en particulier les développements se rapportant aux calculs par simulation Monte Carlo, aux instruments étalons (calorimètres, chambres d'ionisation) et aux références dosimétriques pour la radiothérapie, le radiodiagnostic et la radioprotection.

ABSTRACT Absorbed dose metrology at CEA : the Laboratoire National Henri Becquerel.

The activities related to absorbed dose metrology of the Laboratoire National Henri Becquerel (LNE-LNHB), national metrology laboratory for ionizing radiation, are briefly presented. The results of the main studies made during the last years in that field are reported, in particular developments concerning Monte Carlo calculation, standard instruments (calorimeters, ionization chambers), and dosimetric references for radiotherapy, radiodiagnosis and radiation protection.

Keywords: ionizing radiation / metrology / primary standard / dosimetry

\section{Historique du laboratoire : du Bureau National de Métrologie au LNE}

C'est dans l'environnement du premier réacteur nucléaire français au fort de Chatillon (pile Zoé), que fut créée la Section de Mesures du CEA, ancêtre de l'actuel LNHB. Ce laboratoire affirma son rôle en métrologie de la radioactivité dès le début des années 50 en participant aux premières comparaisons internationales de mesures d'activité et en développant, à partir de 1960, la production de sources étalons de radioactivité. Le laboratoire prit part en 1961 aux premières comparaisons organisées par le BIPM dans le domaine des rayonnements ionisants. Les activités de dosimétrie ont commencé au milieu des années 60. À la création du Bureau National de Métrologie (BNM) en 1969, le laboratoire fut désigné comme laboratoire national de métrologie pour les rayonnements ionisants. Les premiers faisceaux de rayonnement de référence du laboratoire pour la dosimétrie ont été installés à cette époque. Depuis, le laboratoire a été partie prenante des évolutions de l'organisation de la métrologie

\footnotetext{
${ }^{1}$ CEA / LIST / DETECS, Laboratoire National Henri Becquerel, CEA Saclay, 91191 Gif-sur-Yvette cedex, France.
} 
française : transformation du BNM en G. I. P. BNM en 1994, prise en charge en 2001 des références dosimétriques nationales pour les photons $\mathrm{X}$ de basse et moyenne énergie du LCIE et, en 2005, fin du G. I. P. et pilotage de la métrologie nationale par le LNE (Laboratoire national d'essais), rebaptisé Laboratoire national de métrologie et d'essais. Dans ce nouveau cadre, le LNHB a vu son rôle et ses missions confirmés dans les domaines de la radioactivité et de la dosimétrie des photons et particules chargées en devenant l'un des laboratoires nationaux de métrologie fédérés par le LNE, avec l'Institut national de métrologie du CNAM et le SYRTE de l'Observatoire de Paris.

\section{La nécessité d'une dosimétrie primaire}

\subsection{Métrologie : la quête de la précision}

L'utilisation de tout instrument de mesure requiert la connaissance de la relation existant entre l'indication qu'il donne et la grandeur mesurée. Pour un instrument « secondaire », il faut connaître son coefficient d'étalonnage. Cette opération équivaut à comparer l'indication de l'instrument obtenue lors de la mesure à celle obtenue pour un étalon de la grandeur à mesurer. Cet étalon peut avoir été luimême caractérisé par le même procédé au moyen d'un autre étalon, et ainsi de suite. Tous ces étalons, reliés les uns aux autres, forment une chaîne de « traçabilité ». À l'origine, il en faut donc un qui soit réalisé ex nihilo, autrement dit par tout autre moyen qu'un étalonnage dans la grandeur d'intérêt. C'est l'«étalon primaire ». Les laboratoires nationaux de métrologie ont pour mission de réaliser ces étalons. Ceux-ci constituent le premier maillon de la « chaîne » métrologique reliant l'instrument de l'utilisateur aux étalons internationaux. La précision de l'étalonnage d'un instrument de mesure dépend directement de celle de l'étalon primaire. Par ailleurs, chaque maillon ajoutant son incertitude, cette précision est améliorée si la chaîne entre l'étalon primaire et cet instrument est raccourcie.

En dosimétrie, l'étalon primaire est constitué par l'instrument de mesure, qui permet de quantifier la grandeur dosimétrique dans un champ de rayonnement spécifique. Une fois caractérisé, ce champ de rayonnement devient à son tour un étalon de transfert permettant d'étalonner des dosimètres secondaires.

\subsection{Les facteurs de correction : combien sont-ils?}

Le processus de mesure d'une grandeur dosimétrique en un point comporte deux étapes. La première consiste à extraire du volume sensible du détecteur un signal proportionnel à l'énergie qui lui est communiquée par le rayonnement. Cela implique de connaître la relation entre cette énergie et la grandeur effectivement mesurée (charge ou courant électrique, élévation de température, changement de 
concentration d'une espèce chimique, etc.). On a respectivement recours à l'énergie moyenne dépensée pour créer une paire d'ions, $W$, pour les chambres d'ionisation, au rendement calorifique pour les calorimètres, et au rendement radiochimique de production de l'espèce $\mathrm{X}, G(X)$, pour les dosimètres chimiques.

La seconde étape consiste à déduire de cette quantité la grandeur dosimétrique à mesurer. Plusieurs problèmes se posent alors : la quantité mesurée est déterminée dans un volume et non en un point, la nature du volume sensible du détecteur diffère dans la plupart des cas du milieu d'intérêt, tous les autres éléments constituant le détecteur (parois) perturbent le champ de rayonnement qui atteint le volume de détection. Tous ces effets doivent être évalués expérimentalement ou par calcul, et corrigés. Ces corrections sont d'autant plus faibles que les matériaux constitutifs du détecteur ont des numéros atomiques et des densités proches de ceux du milieu d'intérêt.

\subsection{La modélisation numérique : une nécessité}

Les interactions qui interviennent sont complexes. Aussi la détermination expérimentale des facteurs de correction n'est pas toujours possible. De même, le calcul analytique reposant sur des hypothèses simplificatrices peut conduire à des approximations excessives. C'est pourquoi le recours au calcul de ces facteurs par des codes de simulation Monte Carlo d'interactions rayonnement-matière s'est progressivement imposé depuis le début des années 80 dans la communauté des métrologues. Cela ne s'est pas fait sans réticences, notamment à cause de la difficulté d'évaluer l'incertitude de type B résultant des modèles et des bases de données de section efficace utilisés. Compte tenu des incertitudes requises pour les applications métrologiques (incertitude de type A ne devant pas dépasser 0,1\%), la pratique de ces calculs a longtemps été limitée par la puissance des ordinateurs disponibles dans les laboratoires.

Au LNHB, l'utilisation de ces codes a commencé par le calcul de facteurs de correction des interstices de vide du calorimètre en graphite à l'aide des codes EGS 4 et ITS à la fin des années 80 . La première utilisation métrologique du code PENELOPE a été réalisée au LNHB, en collaboration avec son concepteur F. Salvat, à l'occasion de la thèse de J. Mazurier portant sur l'établissement des références de dose absorbée dans l'eau pour les photons X de l'accélérateur médical Saturne 43 du laboratoire (Mazurier, 1999; Mazurier et al., 1999 ; Mazurier et al., 2001). Dans ce cadre a été effectuée la première modélisation de la tête d'un accélérateur médical. Les profils de dose et rendements en profondeur ont été également calculés, ainsi que certains facteurs de correction. Les résultats ont été comparés à ceux obtenus à l'aide d'EGS4 mais aussi aux résultats expérimentaux, montrant un bon accord. Ces travaux ont ouvert la voie à de très nombreuses études similaires sur d'autres LINAC. Le LNHB a, quant à lui, 
poursuivi ce travail pour les faisceaux d'électrons et s'est engagé plus récemment sur la voie de la validation des codes de calculs aux interfaces des milieux hétérogènes (Blazy et al., 2006).

\section{Les techniques de dosimétrie primaire}

\subsection{L'ionométrie par chambres à ionisation : un vaste domaine d'utilisation}

La technique de mesure par chambre d'ionisation joue un rôle prépondérant en dosimétrie, en raison notamment de sa sensibilité et de sa précision, qualités mises à profit dès les découvertes historiques sur les rayons $\mathrm{X}$ et la radioactivité. L'utilisation de ces chambres en tant qu'étalons primaires nécessite de connaître la masse d'air présente dans le volume sensible. Elle implique également la mesure directe de l'intensité du courant d'ionisation ou de la charge. Il faut également connaître la valeur de $W$.

Ces chambres d'ionisation sont très diverses, selon leur fonction, la grandeur mesurée, la nature et l'énergie du rayonnement :

- chambres étalons à paroi d'air pour la mesure du kerma dans l'air pour les faisceaux $\mathrm{X}$ de basse et moyenne énergie,

- chambre à extrapolation pour la mesure de la dose absorbée dans le tissu dans les champs de rayonnement bêta,

- chambres étalons à cavité d'air à paroi de graphite pour la mesure du kerma dans l'air dans les faisceaux de cobalt-60 et césium-137.

Afin de consolider les références établies avec la chambre à cavité étalon réalisée par le laboratoire au milieu des années 80 , une nouvelle série de chambres de ce type a été conçue, et est en cours de tests (Fig. 1). Des chambres à cavité sont également utilisées comme instruments de transfert dans l'établissement de références dosimétriques. Il n'est pas nécessaire dans ce cas de connaître le volume de la cavité et $W$. Par exemple, la dose absorbée dans l'eau dans les faisceaux de photons de haute énergie est dérivée de celle dans le graphite mesurée par calorimétrie par un transfert de milieu, qui peut s'effectuer à l'aide de chambres d'ionisation de petit volume de type radiothérapie.

\subsection{Le calorimètre en graphite et le calorimètre à eau : avantages et inconvénients}

C'est la technique permettant l'accès le plus direct à l'énergie communiquée par le rayonnement à la matière, donc à la dose absorbée, car la dégradation ultime de cette énergie conduit à une simple augmentation de l'agitation thermique du 


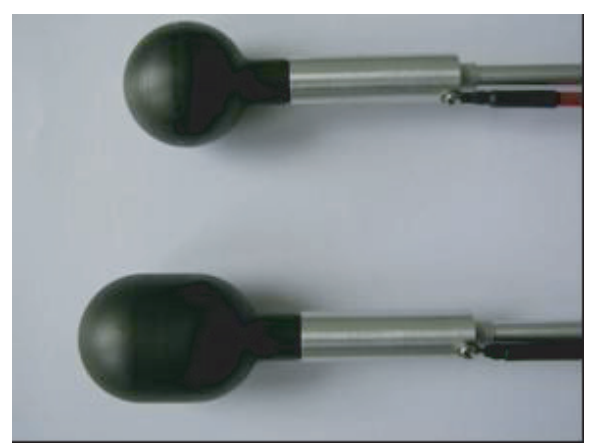

Figure 1 - Chambres étalons à cavité pour la mesure du kerma dans l'air. Air kerma standard cavity ionization chambers.

milieu. La quantité de chaleur mesurée n'est cependant rigoureusement égale à l'énergie communiquée que si aucun défaut de chaleur ne se manifeste dans le matériau considéré. L'application de cette technique est principalement limitée par sa faible sensibilité, de l'ordre du mK/Gy.

Le calorimètre solide est constitué d'un bloc du matériau de référence (graphite ou équivalent-tissus), au sein duquel un absorbeur (élément sensible) de même composition et de masse de l'ordre d'un gramme, est isolé thermiquement du reste de l'instrument par une succession d'interstices de vide et d'écrans. Dans cet absorbeur, la quantité de chaleur résultant de l'irradiation est mesurée avec une thermistance constituant l'une des branches d'un pont de Wheatstone. L'étalonnage du système est effectué en injectant une quantité de chaleur connue par effet Joule. Ce mode de fonctionnement est dit « quasi-adiabatique ».

Le LNHB a acquis une expérience de plus de trente ans dans ce domaine. Les deux calorimètres étalons primaires actuellement opérationnels sont l'un en graphite pour les photons et électrons de haute énergie, l'autre en matériau équivalent-tissus («Shonka ») pour les neutrons et les protons de haute énergie utilisés en radiothérapie. Le rendement calorifique du graphite est pris égal à 1 . Ce n'est pas le cas du matériau Shonka, pour lequel un facteur de correction de quelques pourcents doit être appliqué. Un nouveau calorimètre en graphite a été construit au laboratoire au cours de l'année passée pour remplacer le calorimètre étalon actuel, en service depuis 20 ans et à l'origine de toutes les références actuelles de dose absorbée pour les faisceaux de photons du laboratoire (Fig. 2). 

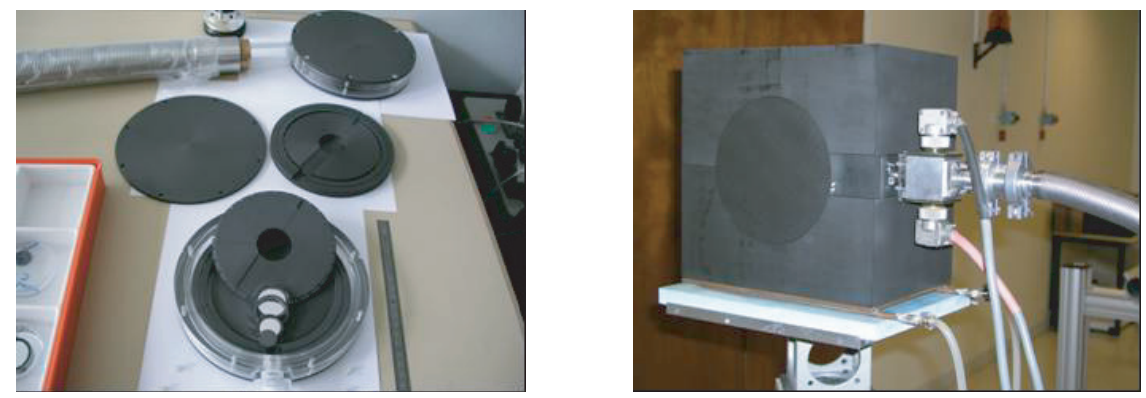

Figure 2 - Calorimètre en graphite du LNHB en cours d'assemblage (à gauche), et calorimètre GR8 inséré dans le fantôme de graphite (à droite).

LNHB graphite calorimeter in construction (left) and calorimeter GR8 inserted in the graphite phantom (right).

Même si les performances du calorimètre graphite étaient déjà excellentes, un nouveau mode de fonctionnement, dit «à température constante », a été mis au point ces dernières années au LNHB (Daures et al., 2005). Le principe consiste à maintenir l'écran à une température fixe et à dissiper dans l'absorbeur une puissance électrique par effet Joule permettant d'atteindre un équilibre thermique. Lors de l'irradiation, la puissance nécessaire au maintien de cet équilibre est réduite proportionnellement à l'énergie déposée par les rayonnements. La valeur du débit de dose est proportionnelle à la différence des puissances électriques en l'absence et en présence d'irradiation. Comparés sur un faisceau de cobalt-60, les résultats obtenus avec les deux modes sont cohérents et s'écartent de moins de $0,04 \%$, l'incertitude de lecture de type A associée au mode à température constante $(0,03 \%)$ étant significativement plus faible que celle du mode quasi adiabatique $(0,06 \%)$.

L'intérêt de la calorimétrie dans l'eau est de pouvoir mesurer la dose absorbée dans l'eau, grandeur de référence en radiothérapie, de la façon la plus directe possible. La mesure de l'élévation de température est également effectuée par l'intermédiaire d'une thermistance constituant l'une des branches d'un pont de Wheatstone. Le défi de cette technique est de pouvoir mesurer un changement local de température qui soit représentatif de la dose absorbée localement : il faut minimiser les phénomènes de convection et stabiliser, ou rendre négligeable, le défaut de chaleur. La mesure de la dose absorbée dans l'eau par ce moyen est complémentaire de celle obtenue à partir de la dose absorbée dans le graphite. Les deux techniques de calorimétrie doivent donc être mises en œuvre au sein d'un même laboratoire de métrologie primaire. 


\subsection{La dosimétrie chimique : le dosimètre de Fricke}

Dans un milieu irradié, les ionisations et excitations atomiques et moléculaires initiales provoquent des modifications chimiques qui peuvent, dans certains cas, permettre une mesure dosimétrique. Le système chimique le plus utilisé, et mis en oeuvre depuis de nombreuses années au LNHB, est le dosimètre de Fricke basé sur l'oxydation d'une solution aqueuse acide de sulfate ferreux. L'entité considérée est l'ion ferrique résultant de cette oxydation, dont la concentration est mesurée par spectrophotométrie. Ce système présente l'avantage d'être quasi-identique à l'eau. Sa sensibilité est cependant faible : pour une utilisation métrologique, la dose minimale acceptable est de $20 \mathrm{~Gy}$. En raison de la très bonne répétabilité obtenue au laboratoire, ce dosimètre est utilisé essentiellement de façon relative, comme instrument de transfert pour dériver la dose absorbée dans l'eau de celle dans le graphite, de façon identique aux chambres d'ionisation (Chauvenet et al., 1997). La connaissance du rendement radiochimique $G$ n'est pas nécessaire dans ce cas. Tout au plus faut-il connaître sa variation éventuelle en fonction de l'énergie du rayonnement et de sa nature, lorsqu'une mesure relative est effectuée entre deux rayonnements de natures et/ou d'énergies différentes.

\section{La dosimétrie de référence pour la radioprotection}

Les grandeurs de protection, comme la dose efficace et la dose équivalente, qui servent entre autres à établir les limites d'exposition aux rayonnements ionisants, ne sont pas mesurables du fait même de leur définition et de leur mode de calcul, fondés sur la connaissance du dépôt d'énergie dans un fantôme anthropomorphique. Le concept de grandeur opérationnelle mesurable est apparu pour permettre une estimation des grandeurs de protection. C'est ainsi qu'ont été définis les équivalents de dose. Leurs valeurs théoriques sont déterminées au sein de fantômes de forme simple (sphère, cube, rondin), constitués d'un matériau équivalent au tissu « mou » (dit matériau ICRU), au moyen de codes Monte Carlo de calculs de transport du rayonnement. Ces calculs permettent d'établir des coefficients de passage des grandeurs primaires (dites aussi physiques) vers les grandeurs opérationnelles. De fait, un dosimètre ne pouvant qu'enregistrer des événements relatifs aux interactions physiques ayant lieu en son sein, il n'est pas possible de disposer d'un dosimètre absolu qui fournisse une information en termes d'équivalent de dose. La métrologie de référence pour la radioprotection s'appuie donc sur les grandeurs dites « primaires ». Dans la pratique, on utilise :

$>$ pour les électrons et les particules lourdes chargées, la dose absorbée dans le tissu mou à $0,07 \mathrm{~mm}$ de profondeur, $D_{\mathrm{t}}(0,07)$. Le mode d'interaction coulombien, en excluant la production de rayonnements de freinage aux 
énergies rencontrées en radioprotection, conduit à une absorption locale de l'énergie déposée par le rayonnement. Notons que certains pays (Royaume Uni, Suède, Irlande) utilisent aussi dans ce cas la dose absorbée dans l'air ; pour les photons, le kerma dans l'air, $K_{\text {air }}$.

\section{1. Étalons du LNHB pour les photons du cobalt-60 et du césium-137}

Les instruments étalons primaires utilisés pour la caractérisation des faisceaux de photons de référence de cobalt-60 et de césium-137 en kerma dans l'air sont des chambres d'ionisation à cavité ouvertes en graphite fabriquées selon les plans élaborés par le LNHB (Leitner et al., 1997).

\section{2. Étalons du LNHB pour le rayonnement bêta}

La dose absorbée dans les tissus sous $7 \mathrm{mg} . \mathrm{cm}^{2}$ est mesurée au moyen d'une chambre d'ionisation étalon à extrapolation. L'épaisseur de la fenêtre d'entrée de la chambre est fixée à $7 \mathrm{mg} . \mathrm{cm}^{2}$. Des mesures successives sont effectuées en faisant varier l'épaisseur du volume d'air de collection, en maintenant le champ électrique constant (Lecante et al., 2004). Les valeurs de courant obtenues permettent par ajustement de tracer une droite, ou une fonction polynomiale, en fonction de l'épaisseur de la cavité. C'est la valeur de la pente de cette droite, ou, pour une fonction polynomiale, la pente à l'origine (épaisseur nulle), qui est utilisée pour pouvoir appliquer correctement la relation de Bragg-Gray et en déduire la dose absorbée.

Le LNHB dispose de références pour les champs de rayonnement bêta de strontium/yttrium-90, de krypton-85 (qui s'est substitué au thallium-204 ces dernières années) et de prométhéum-147, par ordre décroissant d'énergie. Les incertitudes associées à ces références dépendent entre autres de l'énergie du rayonnement : plus celle-ci est faible, plus l'influence de paramètres comme la distance de mesure est importante.

\section{La dosimétrie de référence pour le radiodiagnostic}

Les centres français de diagnostic médical doivent faire face à des obligations en termes de contrôle qualité des installations. La mise en place de ces références a pour but de répondre à leurs besoins de raccordement métrologique. Cette action a débuté au sein du BNM en 1996 dans les laboratoires du LCIE avec la mise en place de références nationales en mammographie en termes de kerma dans l'air. L'appareil choisi est un modèle possédant une anode bi-métal molybdène et rhodium et permettant de travailler entre $22 \mathrm{kV}$ et $49 \mathrm{kV}$. Une chambre à parois d'air dédiée à cette installation a été spécialement conçue. 

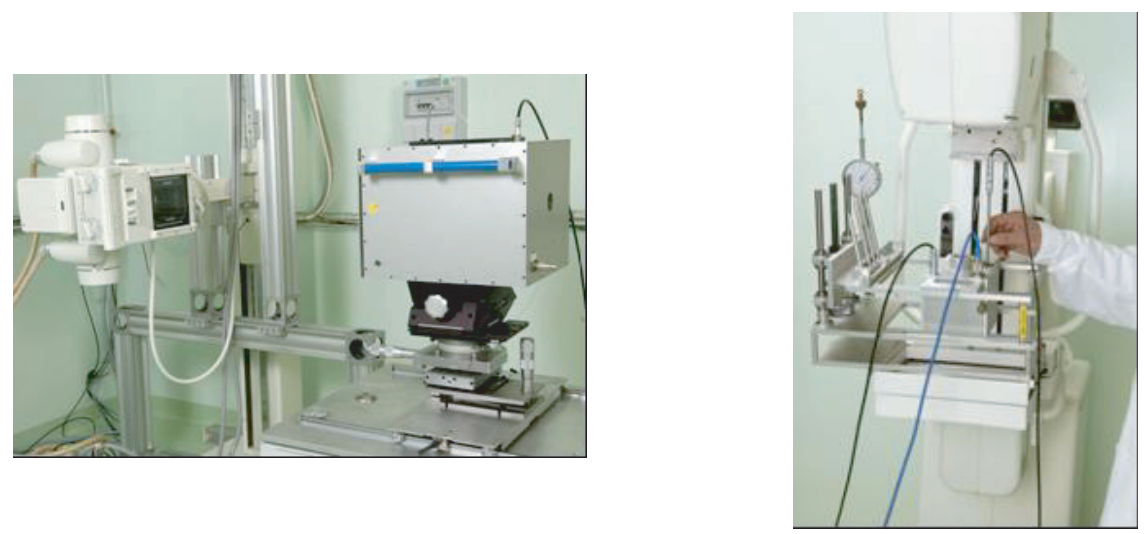

Figure 3-Appareil de radiodiagnostic du LNHB Figure 4 - Mammographe du LNHB et chambre (à gauche) et chambre étalon à paroi d'air associée (à droite). étalon à paroi d'air associée.

Radiodiagnosis device of LNHB (left) and associated standard free-air LNHB mammograph and associated standard free-air ionization chamber. ionization chamber (right).

La mise en place des références nationales en radiodiagnostic médical a débuté en 2000 au LCIE. Elle s'est poursuivie en 2001 sous la responsabilité du LNHB. L'appareil choisi est un modèle travaillant entre 40 et $150 \mathrm{kV}$, avec une anode tournante en tungstène, et pouvant donner des faisceaux « graphie » et « scopie ». Il est représentatif des installations rencontrées en France. L'ensemble des équipements a été transféré dans les locaux du LNHB fin 2003.

Les étalons primaires sont des «chambres d'ionisation à paroi d'air». Ces dosimètres sont adaptés par leurs dimensions à l'énergie des rayons $\mathrm{X}$ émis par les générateurs équipant les appareils de radiodiagnostic $\left(E_{x} \sim 150 \mathrm{keV}\right)$ (Fig. 3) et de mammographie ( $\left.E_{x} \sim 50 \mathrm{keV}\right)$ (Fig. 4).

Les générateurs médicaux délivrent des faisceaux pulsés d'une durée de quelques millisecondes à quelques secondes. Cette caractéristique conduit à étudier les problèmes de recombinaison des charges de manière approfondie ; on constate que pour des durées d'impulsion supérieures à environ $100 \mathrm{~ms}$, les résultats obtenus avec les faisceaux pulsés et continus sont statistiquement équivalents. Les trois principales composantes de l'incertitude sont la reproductibilité de la mesure de la charge, incluant le positionnement du détecteur et le réglage du faisceau $(0,25 \%)$, le volume de détection $(0,20 \%)$ et la valeur de $W_{\text {air }}$ /e $(0,15 \%)$. L'incertitude composée relative élargie est égale à $0,78 \%(k=2)$. On peut noter que le besoin en termes de précision sur la « dose » délivrée aux 
patients pour les examens radiographiques a été estimé à $30 \%$ et $10 \%(k=3)$, respectivement chez l'adulte et l'enfant.

\section{La dosimétrie de référence pour la radiothérapie : un besoin de société}

La dosimétrie de radiothérapie met en oeuvre des programmes de planification des traitements (dits TPS pour «treatment planning system»). Ces programmes simulent la balistique des traitements sur le patient afin de se conformer au mieux aux contours de la tumeur définis par le médecin sur la base des images de diagnostic. Les données d'entrée de ces logiciels sont d'une part des images en trois dimensions du patient reconstituées à partir des images de diagnostic, d'autre part les caractéristiques des faisceaux de rayonnement. Celles-ci sont mesurées au moyen du dosimètre de référence du service de radiothérapie. Celui-ci doit être étalonné de la façon la plus précise possible. On distingue la radiothérapie externe, pour laquelle la source de rayonnement est située à l'extérieur du corps, et la curiethérapie pour laquelle la source de rayonnement est placée dans le corps au plus près de la tumeur.

\subsection{La radiothérapie externe et le programme DELPHES}

Le LNHB disposait déjà d'une source de cobalt-60 de type cobalthérapie caractérisée successivement en kerma dans l'air et, au début des années 1990, en dose absorbée dans l'eau. Cette référence lui permettait d'étalonner les dosimètres de référence des utilisateurs, de façon directe pour les faisceaux de cobalthérapie, et de façon indirecte pour les faisceaux d'accélérateurs médicaux, via les Codes de pratique pour la dosimétrie en radiothérapie, par exemple ceux publiés par l'AIEA. Le développement très rapide du parc d'accélérateurs rendait nécessaire de développer des références dosimétriques auprès d'un accélérateur d'électrons, de préférence de type médical, et de proposer ainsi aux utilisateurs des étalonnages effectués dans des faisceaux dont les qualités soient aussi proches que possible des leurs, avec une incertitude réduite.

Le LNHB a donc lancé le projet DELPHES (Dosimétrie des ELectrons et Photons de Hautes EnergieS) au milieu des années 1990 et s'est équipé en 1996 d'un accélérateur linéaire médical Saturne 43 (G. E.). Cet accélérateur peut produire des faisceaux d'électrons (8 énergies entre $4 \mathrm{MeV}$ et $21 \mathrm{MeV}$ ) et des faisceaux de photons $\mathrm{X}$ de freinage ( 9 qualités entre $4 \mathrm{MV}$ et $25 \mathrm{MV}$ ). La mise en œuvre de cet équipement, associée aux techniques dosimétriques du laboratoire (calorimétrie, dosimétrie chimique et dosimétrie par chambre d'ionisation) a permis de mettre en place des références nationales en termes de dose absorbée 


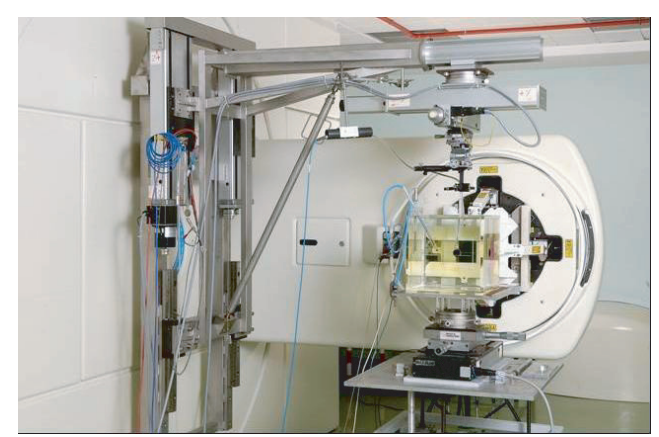

Figure 5 - Programme DELPHES : accélérateur Saturne 43 (G. E.) en configuration «électrons ». On peut voir une chambre d'ionisation dans le fantôme d'eau.

Program DELPHES: Saturne 43 (G. E.) accelerator in “electron” mode. An ionization chamber can be seen in the water phantom.

dans l'eau pour les photons (Shortt et al., 2001) et les électrons de haute énergie et de les transférer aux utilisateurs (Fig. 5).

De manière à répondre au mieux au besoin exprimé, le LNHB réalise non seulement un étalonnage "physique» du dosimètre pour trois qualités de faisceaux (définies en termes de TPR 20,10 ou de $R_{50}$ respectivement pour les photons et les électrons) couvrant l'ensemble du domaine d'utilisation, mais fournit aussi le coefficient d'étalonnage à la qualité de faisceau voulue, obtenu par un calcul d'interpolation.

On admet en général qu'une erreur maximale de $5 \%$ sur la dose délivrée à la tumeur est admissible, dernièrement cette valeur tend à être ramenée à 3,5\%. Ce dernier chiffre n'est que très peu supérieur à l'incertitude (avec un facteur d'élargissement égal à 2) associée aux étalonnages des dosimètres de référence des services de radiothérapie. C'est donc l'ensemble du processus qui conduit à la délivrance d'une dose prescrite à la tumeur qu'il faut améliorer. C'est notamment dans cette perspective que le LNHB s'est impliqué dans la validation des logiciels de TPS, face à l'extension de techniques telles que l'IMRT et l'IGRT. Il est essentiel que cette validation soit faite au meilleur niveau métrologique.

\subsection{La curiethérapie et le programme CIRCE}

La curiethérapie à haut débit de dose constitue une alternative intéressante à la radiothérapie conventionnelle et à la chirurgie pour le traitement de certains cancers (poumon, prostate, etc.). Afin d'assurer la traçabilité des soins, le LNHB a 

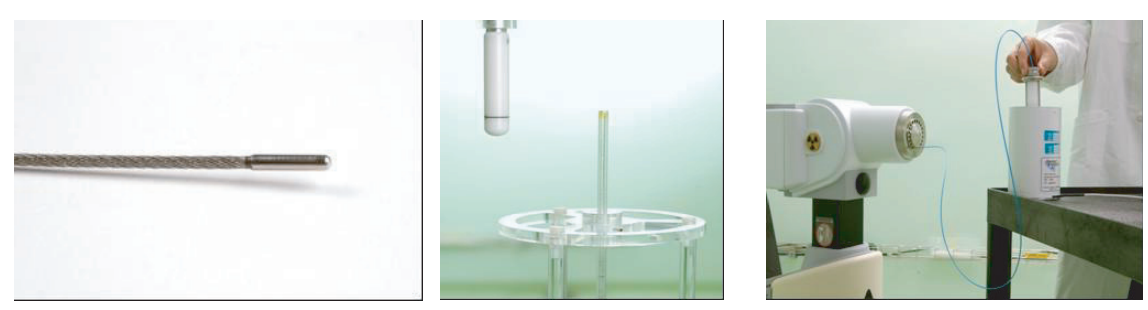

Figure 6-À gauche, source d'iridium-192 au bout de son câble de projection; au centre, le système de mesure par rotation (chambre à cavité à gauche et support de la source en PMMA à droite); à droite le projecteur de source relié à une chambre d'ionisation à puits pour le transfert de la référence.

On the left, iridium-192 source at the end of its loading wire; in the middle, rotating measuring system (standard ionization chamber and PMMA source holder); on the right, remote source afterloader coupled with the well-type ionization chamber for calibration.

été chargé de développer un étalon dosimétrique français pour la caractérisation des sources d'iridium-192 utilisées pour ce type d'application. Ainsi en 2001, le programme CIRCÉ (Curiethérapie, Iridium, Référence, Contrôles et Étalonnages) a été initié. Le dispositif expérimental a été mis en place avec succès durant l'année 2002.

La grandeur d'intérêt est le débit de kerma dans l'air de référence (Gy/h à $1 \mathrm{~m})$. L'étalon est une chambre d'ionisation à cavité préalablement étalonnée dans des faisceaux X de moyenne énergie et de photons $\gamma$ du césium-137. Elle est choisie de manière à avoir une réponse variant peu en fonction de l'énergie. Pour la mesure, elle est placée à une distance variant de 10 à $15 \mathrm{~cm}$ de la source. À la réception d'une nouvelle source, ce dosimètre étalon est utilisé pour caractériser la source en termes de débit de kerma dans l'air de référence. Le dispositif de mesure fait appel à un système de positionnement précis à $50 \mu \mathrm{m}$ près, qui permet d'assurer un niveau d'incertitude sur le résultat final de $0,6 \%(\mathrm{k}=1)$. Le détecteur tourne autour de la source, permettant des mesures selon des angles différents (Fig. 6). Après chaque série de mesure, la position de la source est ajustée de sorte que le détecteur mesure le même kerma de référence quel que soit l'angle. La source ainsi caractérisée est ensuite utilisée pour étalonner les dosimètres secondaires des utilisateurs (chambres d'ionisation à puits).

Une comparaison internationale a été réalisée avec le laboratoire de référence des États-Unis au cours de l'année 2003 (Douysset et al., 2005). L'écart constaté entre les deux références est inférieur à $0,3 \%$, écart au demeurant inscrit dans les incertitudes de mesure. La référence ainsi établie était la première de ce type en Europe. Il a ainsi été possible de procéder aux premiers étalonnages sous accréditation COFRAC à la fin du premier trimestre de l'année 2004. D'autres 


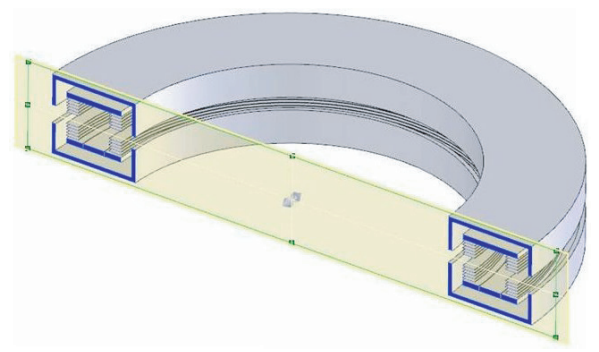

Figure 7 - Vue artistique en coupe de la moitié de la chambre d'ionisation à paroi d'air toroüdale. La source est placée au centre du cercle.

Artist view (vertical section) of a half of the toroidal free-air ionization chamber. The source is placed at the centre.

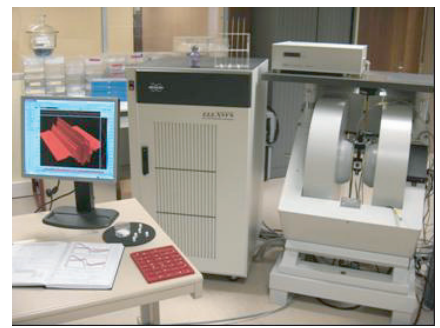

Figure 8-Spectromètre RPE : sur la gauche, bâti des alimentations; sur la droite, électroaimant et cavité; au-dessus, le générateur hyperfréquence (9-10 GHz, diode gun).

ESR spectrometer: on the left, power pack; on the right, electro-magnet and cavity; above, $\mathrm{HF}$ generator $(9-10 \mathrm{GHz}$, diode gun).

laboratoires européens de métrologie ont depuis mis au point leur référence dosimétrique. Ainsi, une comparaison bilatérale a été effectuée avec le NPL en 2005, les résultats préliminaires ont montré un très bon accord.

En 2006, le LNHB a entrepris de mettre au point une référence nationale pour les implants prostatiques d'iode-125. Le détecteur est d'une conception innovante, il s'agit d'une chambre à paroi d'air de forme toroïdale (Fig. 7). La source est positionnée au centre de ce tore. Les premiers essais ont commencé à la fin de l'année passée.

\section{La dosimétrie par Résonance Paramagnétique Électronique (RPE) : de la métrologie à la datation}

Le LNHB utilise l'alanine comme dosimètre relatif et la spectrométrie par RPE pour sa lecture (Fig. 8). L'alanine est un acide aminé dont les spécificités physicochimiques permettent de mesurer une large gamme de doses (de quelques grays à $100 \mathrm{kGy}$ ). Cela de manière reproductible, avec une faible incertitude et un faible fading, plus ou moins indépendamment du débit de dose, selon la forme chimique adoptée. Au LNHB, cette méthode est appliquée en routine pour les fortes doses (100 Gy - $100 \mathrm{kGy}$ ) et pour les faibles doses (1-10 Gy) avec une incertitude relative globale inférieure à 1,5\% (Dolo et al., 2005a, 2005b). Dans le cadre des 
applications médicales, cette technique peut être une alternative aux dosimètres thermo-luminescents dans le cadre de tests inter-laboratoires.

\section{Histoire inachevée : $W$ est-il une constante universelle ?}

\subsection{Contexte}

La genèse de calcul de $W$ trouve son origine dans la modélisation d'une chambre à extrapolation pour l'établissement des références de dosimétrie bêta. Le problème posé, toujours d'actualité, est de comprendre, sur des bases physiques, le comportement du courant généré dans ce type de chambre lorsque la distance entre électrodes tend vers zéro ou vers des valeurs très faibles devant le libre parcours moyens des électrons incidents. Il a donc paru intéressant de demander à P. Ségur (CPAT de Toulouse), spécialiste des gaz faiblement ionisés, une étude sur ce sujet.

Un certain nombre de différences existent dans les conditions de définition, de mesures et d'utilisation de $W$. En premier lieu, $W$ est défini dans un milieu homogène infini sans champ électrique, alors qu'il est utilisé pour des chambres à ionisation de dimensions finies au sein desquelles il existe un champ électrique suffisant pour minimiser les recombinaisons. Cette constatation posée, on est en droit de se demander si le bilan global est nul ou non, c'est-à-dire si l'application d'un champ électrique modifie la probabilité de créer une paire d'ions et donc $W$.

\subsection{Wen milieu infini sans champ électrique}

En milieu « infini », $W$ est égal au rapport de l'énergie cinétique initiale au nombre total d'ionisations créées lorsque l'énergie est complètement dissipée dans le gaz. La figure 9 montre l'évolution de $W$ en fonction de l'énergie cinétique initiale des électrons comprise entre $10 \mathrm{eV}$ et $100 \mathrm{keV}$, par rapport à la valeur proposée par l'ICRU. L'évolution montre qu'un électron incident ayant une énergie inférieure au seuil d'ionisation est incapable d'ioniser, la valeur de $W$ tend vers l'infini.

\subsection{Wen milieu fini avec champ électrique}

Dans le cas d'un milieu fini, puisque les électrons ne dissipent pas complètement leur énergie dans le milieu, on doit maintenant définir $W$ comme le rapport entre l'énergie effectivement perdue dans le milieu et le nombre d'ionisations constaté dans ce milieu. Les valeurs de $W$ en milieu fini sont inférieures à la valeur calculée en milieu infini. 


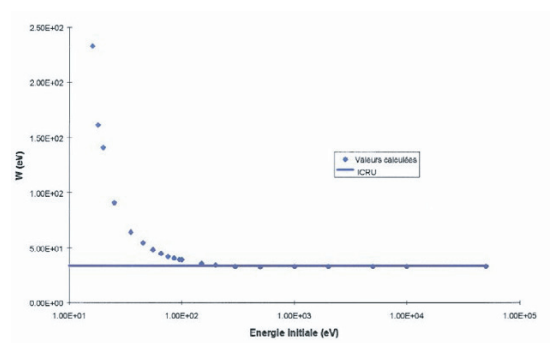

Figure 9 - Calcul de W en milieu infini dans l'air. $W$ calculation in an air infinite volume

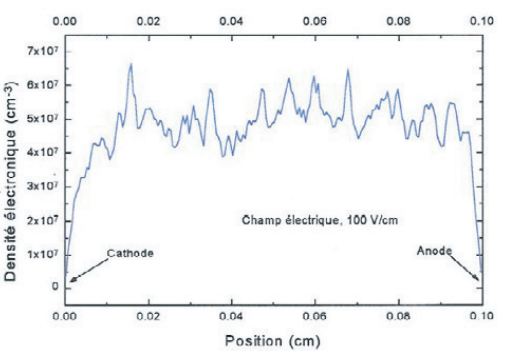

Figure 11-Variation spatiale de la densité électronique en présence de champ électrique.

Spatial variation of the electronic density under applied electric field.

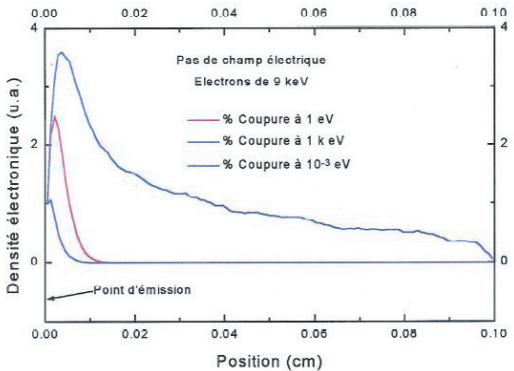

Figure 10-Variation spatiale de la densité électronique en l'absence de champ électrique.

Spatial variation of the electronic density without an applied electric field.

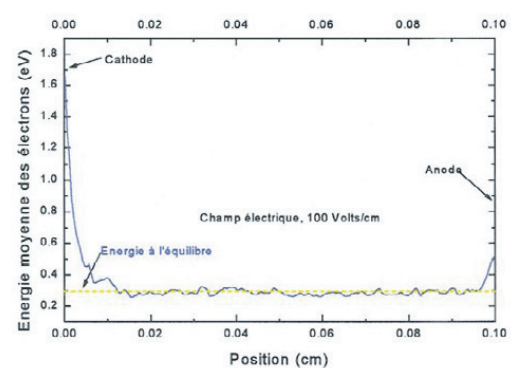

Figure 12-Variation de l'énergie moyenne des électrons en présence d'un champ électrique.

Mean electron energy variation under applied electric field.

Ceci est confirmé, figures 10 et 11, par la forme de la densité électronique en l'absence ou en présence d'un champ électrique (énergie initiale des électrons $9 \mathrm{keV}$, distance inter-électrodes $1 \mathrm{~mm}$ ). On voit que la distribution des électrons est fortement dépendante de l'énergie de coupure et que plus l'énergie de coupure est faible, plus les électrons parcourent une distance importante, ne perdant à chaque collision qu'une très faible quantité d'énergie.

En présence d'un champ électrique, on pourrait a priori croire à des résultats similaires. La figure 12 montre qu'il n'en est rien et que la densité électronique devient très rapidement constante dès que l'on s'éloigne des électrodes, signifiant qu'un régime d'équilibre s'installe en présence d'un champ 
électrique. On montre ainsi que si $W$ dépend des conditions dans lesquelles il est mesuré et donc utilisé, sa variation semble faible. Cependant les calculs réalisés ne sont pas encore assez précis pour trancher...

\section{RÉFÉRENCES}

Blazy L., Baltès D., Bordy J.M., Cutarella D., Delaunay F., Gouriou J., Leroy E., Ostrowsky A., Beaumont S. (2006) Comparison of PENELOPE Monte Carlo dose calculation with Fricke and ionization chambers measurements in heterogeneous phantoms (18 MeV electron and $12 \mathrm{MV}$ photon beams), Phys. Med. Biol. 51, 5951-5965.

Chauvenet B., Baltès D., Delaunay F. (1997) Comparison of graphite-to-water absorbed-dose transfers for ${ }^{60}$ Co photon beams using ionometry and Fricke dosimetry, Phys. Med. Biol. 42, 2053-2063.

Daures J., Ostrowsky A. (2005) New constant-temperature operating mode for graphite calorimeter at LNE-LNHB, Phys. Med. Biol. 50, 4035-4052.

Dolo J.M., Feaugas V. (2005) Analysis of parameters that influence the amplitude of the ESR/alanine signal after irradiation, Appl. Rad. Isotopes 62, 273-279.

Dolo J.M., Moignau F. (2005) Use of the entire spectrum of irradiated alanine for dosimetry, Appl. Rad. Isotopes 62, 281.

Douysset G., Gouriou J., Delaunay F., DeWerd L., Stump K., Mickal J. (2005) Comparison of dosimetric standards of USA and France for HDR brachytherapy, Phys. Med. Biol. 50, 19611978.

Lecante C., Uryaev I., Villevalde N., Rumyantseva E., Chauvenet B. (2004) Comparison of extrapolation chamber measurements of the absorbed dose rate in beta radiation, Metrologia $\mathbf{4 1}$ 06009.

Leitner A., Witzani J., Boutillon M., Allisy-Roberts P., Delaunay F., Leroy E., Lamperti P., Strachotinsky C., Csete I. (1997) International comparisons of air kerma standards in ${ }^{137} \mathrm{Cs}$ gamma radiation, Metrologia 34, 169-175.

Mazurier J. (1999) Adaptation du code Monte Carlo PENELOPE pour la métrologie de la dose absorbée : caractérisation des faisceaux de photons $\mathrm{X}$ de haute énergie et calcul de facteurs de correction de dosimètres de référence, Thèse de 1'université Paul Sabatier de Toulouse, $\mathrm{n}^{\circ}$ d'ordre : 3402, Rapport CEA-R-5879.

Mazurier J., Salvat F., Chauvenet B., Barthe J. (1999) Simulation of photon beams from a Saturne 43 accelerator using the code PENELOPE, Phys. Medica XV, N. 3, 101-110.

Mazurier J., Gouriou J., Chauvenet B., Barthe J. (2001) Calculation of perturbation correction factors for some reference dosimeters in high-energy photon beams with the Monte Carlo code PENELOPE, Phys. Med. Biol. 46, 1707-1717.

Shortt K., Ross C., Seuntjens J., Delaunay F., Ostrowsky A., Gross P., Leroy E. (2001) Comparison of dosimetric standards of Canada and France for photons at 60Co and higher energies, Phys. Med. Biol. 46, 2119-2142. 